\title{
Common Fixed Point Theorems of Multi-Valued Maps in Ultra Metric Space
}

\author{
Qiulin Wang, Meimei Song* \\ College of Science, Tianjin University of Technology, Tianjin, China \\ Email: *songmeimei@tjut.edu.cn
}

Received November 30, 2012; revised January 9, 2013; accepted January 16, 2013

\begin{abstract}
We establish some results on coincidence and common fixed point for a two pair of multi-valued and single-valued maps in ultra metric spaces.
\end{abstract}

Keywords: Multi-Valued Maps; Coincidence Point; Common Fixed Point

\section{Introduction}

Roovij in [1] introduced the concept of ultra metric space. Later, C. Petalas, F. Vidalis [2] and Ljiljana Gajic [3] studied fixed point theorems of contractive type maps on a spherically complete ultra metric spaces which are generalizations of the Banach fixed point theorems. In [4] K. P. R. Rao, G. N. V. Kishore and T. Ranga Rao obtained two coincidence point theorems for three or four self maps in ultra metric space.

J. Kubiaczyk and A. N. Mostafa [5] extend the fixed point theorems from the single-valued maps to the setvalued contractive maps. Then Gajic [6] gave some generalizations of the result of [3]. Again, Rao [7] proved some common fixed point theorems for a pair of maps of Jungck type on a spherically complete ultra metric space.

In this article, we are going to establish some results on coincidence and common fixed point for two pair of multi-valued and single-valued maps in ultra metric spaces.

\section{Basic Concept}

First we introducing a notation.

Let $C(X)$ denote the class of all non empty compact subsets of $X$. For $A, B \in C(X)$, the Hausdorff metric is defined as

$$
H(A, B)=\max \left\{\sup _{x \in A} d(x, B), \sup _{y \in B} d(y, A)\right\}
$$

where $d(x, A)=\inf \{d(x, a): a \in A\}$.

The following definitions will be used later.

Definition 2.1 ([1]) Let $(X, d)$ be a metric space. If the metric $d$ satisfies strong triangle inequality

*Corresponding author.

$$
d(x, y) \leq \max \{d(x, z), d(z, y)\}, \forall x, y, z \in X
$$

Then $d$ is called an ultra metric on $X$ and $(X, d)$ is called an ultra metric space.

Example. Let $X \neq \theta, d(x, y)=\left\{\begin{array}{l}0, x=y \\ 1, x \neq y\end{array}\right.$, then $(X, d)$ is a ultra metric space.

Definition 2.2 ([1]) An ultra metric space is said to be spherically complete if every shrinking collection of balls in $X$ has a non empty intersection.

Definition 2.3 An element $x \in X$ is said to be a coincidence point of $T: X \rightarrow C(X)$ and $f: X \rightarrow X$ if $f x \in T x$. We denote

$$
C(f, T)=\{x \in X \mid f x \in T x\}
$$

the set of coincidence points of $T$ and $f$.

Definition 2.4 ([7]) Let $(X, d)$ be an ultra metric space, $f: X \rightarrow X$ and $T: X \rightarrow C(X) . T$ and $f$ are said to be coincidentally commuting at $z \in X$ if $f z \in T z$ implies $f T z \subseteq T f z$.

Definition 2.5 ([8]) An element $x \in X$ is a common fixed point of $T, S: X \rightarrow C(X)$ and $f: X \rightarrow X$ if $x=f x \in T x \cap S x$.

\section{Main Results}

The following results are the main result of this paper.

Theorem 3.1 Let $(X, d)$ be an ultra metric space. Let $T, S: X \rightarrow C(X)$ be a pair of multi-valued maps and $f, g: X \rightarrow X$ a pair of single-valued maps satisfying

(a) $f g(X)$ is spherically complete;

(b) $H(S x, T y)<\max \{d(f x, g y), d(f x, S x), d(g y, T y)\}$

for all $x, y \in X$, with $f x \neq g y$; 
(c) $f S=S f, f g=g f, f T=T f, g S=S g, g T=T g$, $S T=T S$;

(d) $S(X) \subseteq f(X), T(X) \subseteq g(X)$.

Then there exist point $u$ and $v$ in $X$, such that $f u \in S u, g v \in T v, f u=g v, S u=T v$.

\section{Proof. Let}

$$
B_{a}=(f g a ; \max \{d(f g a, S g a), d(f g a, T f a)\})
$$

denote the closed sphere with centered $f g a$ and radius

$$
\max \{d(f g a, S g a), d(f g a, T f a)\} .
$$

Let $A$ be the collection of all the spheres for all $a \in f g(X)$.

Then the relation

$$
B_{a} \leq B_{b} \text { if } B_{b} \subseteq B_{a}
$$

is a partial order on $A$.

Consider a totally ordered sub family $A_{1}$ of $A$.
Since $f g(X)$ is spherically complete, we have

$$
\bigcap_{B_{a} \in A_{1}} B_{a}=B \neq \varnothing
$$

Let $f g b \in B$ where $b \in f g(X)$ and $B_{a} \in A_{1}$.

Then $f g b \in B_{a}$. Hence

$$
d(f g b, f g a) \leq \max \{d(\text { fga }, \text { Sga }), d(\text { fga }, T f a)\}
$$

If $a=b$ then $B_{a}=B_{b}$. Assume that $a \neq b$.

Let $\forall x \in B_{b}$, then

$$
d(x, f g b) \leq \max \{d(f g b, S g b), d(f g b, T f b)\}
$$

Since Sga is nonempty compact set, then $\exists p \in S g a$ such that

$$
d(\text { fga, Sga })=d(\text { fga }, p) ;
$$

$T f a$ is a nonempty compact set, then $\exists q \in T f a$ such that $d(f g a, T f a)=d(f g a, q)$.

$$
\begin{aligned}
& \max \{d(f g b, S g b), d(f g b, T f b)\}=\max \left\{\inf _{c \in S g b} d(f g b, c), \inf _{d \in T f b} d(f g b, d)\right\} \\
& \leq \max \left\{d(f g b, f g a), d(f g a, q), \inf _{c \in S g b} d(q, c), d(f g b, f g a), d(f g a, p), \inf _{d \in T f b} d(p, d)\right\} \\
& \leq \max \{d(f g b, f g a), d(f g a, T f a), H(T f a, S g b), d(f g b, f g a), d(f g a, S g a), H(S g a, T f b)\} \\
& <\max \{d(f g b, f g a), d(f g a, T f a), d(f g a, S g a), \max \{d(f g b, f g a), d(g f a, T f a), d(f g b, S g b)\}, \\
& \quad \max \{d(f g a, f g b), d(f g a, S g a), d(g f b, T f b)\}\} \\
& \leq \max \{d(f g a, T f a), d(f g a, S g a)\}
\end{aligned}
$$

from (a) (b) and Equation (1)

Now

$$
\begin{aligned}
d(x, f g a) & \leq \max \{d(x, f g b), d(f g b, f g a)\} \\
& \leq \max \{d(\text { fga }, \text { Sga }), d(\text { fga }, \text { Tfa })\}
\end{aligned}
$$

So $x \in B_{a}$, we have just proved that $B_{b} \subseteq B_{a}$ for every $B_{a} \in A_{1}$. Thus $B_{b}$ is an upper bound in $A$ for the family $A_{1}$ and hence by Zorn's Lemma, there is a maximal element in $A$, say $B_{z}, z \in f g(X)$. There exists $w \in X$ such that $z=f g w$.
Suppose

$$
f(g f g w) \notin S(g f g w), g(f f g w) \notin T(f f g w) .
$$

Since Sgfgw,Tffgw are nonempty compact sets, then $\exists k \in S g f g w, t \in T f f g w$ such that

$$
\begin{gathered}
d(\text { fgfgw, Sgfgw })=d(\text { fgfgw }, k), \\
d(f g f g w, T f f g w)=d(f g f g w, t)
\end{gathered}
$$

From (b), (c) and Equation (2), we have

$$
\begin{aligned}
& d(\text { Sgfgw, TSfgw })=\inf _{e \in T S f g} d(g S f g w, e) \leq \max \left\{d(S g f g w, f g f g w), d(f g f g w, k), \inf _{e \in T S f g w} d(k, e)\right\} \\
& \leq \max \{d(\text { Sgfgw, fgfgw }), H(\text { Sgfgw, TSfgw })\} \\
& <\max \{d(\text { Sgfgw, fgfgw }), \max \{d(f g f g w, f S f g w), d(f g f g w, S g f g w), d(g S f g w, T S f g w)\}\} \\
& =d(f g f g w, S g f g w) \\
& d(T f f g w, S T f g w)=\inf _{h \in S T g w} d(f T f g w, h) \leq \max \left\{d(T f f g w, f g f g w), d(f g f g w, t), \inf _{h \in S T g w} d(t, h)\right\} \\
& \leq \max \{d(T f f g w, f g f g w), H(T f f g w, S T f g w)\} \\
& <\max \{d(T f f g w, f g f g w),\{\max d(f T f g w, g f f g w), d(f T f g w, S T f g w), d(g f f g w, T f f g w)\}\}=d(T f f g w, f g f g w)
\end{aligned}
$$


From (b), (c) and Equations (2)-(5)

$$
\begin{aligned}
& d(f g g S w, S g g S w)=\inf _{m \in S g g S w} d(f g g S w, m) \\
& \leq \max \left\{d(f g g S w, T S f g w), d(T S f g w, T f f g w), d(T f f g w, f g f g w), d(f g f g w, t), \inf _{m \in S g g S w} d(t, m)\right\} \\
& \leq \max \{d(f g f g w, S g f g w), d(\text { fgfgw, Tffgw }), H(T f f g w, S g g S w)\} \\
& <\max \{d(f g f g w, S g f g w), d(f g f g w, T f f g w), \max \{d(f g g S w, g f f g w), d(f g g S w, S g g S w), d(g f f g w, T f f g w)\}\} \\
& \leq \max \{d(\text { fgfgw, Sgfgw }), d(\text { fgfgw,Tffgw })\} \\
& d(f g f T w, T f f T w)=\inf _{n \in T f f w} d(f g f T w, n) \\
& \leq \max \left\{d(\text { fgfTw, STfgw }), d(\text { STfgw, Sgfgw }), d(\text { Sgfgw, fgfgw }), d(\text { fgfgw }, k), \inf _{n \in I f T w} d(k, n)\right\} \\
& \leq \max \{d(\text { fgfgw, Tffgw }), d(\text { fgfgw, Sgfgw }), H(\text { Sgfgw,TffTw })\} \\
& <\max \{d(\text { fgfgw,Tffgw }), d(\text { fgfgw, Sgfgw }), \max \{d(\text { fgfgw, gffTw }), d(\text { fgfgw, Sgfgw }), d(g f f T w, T f f T w)\} \\
& \leq \max \{d(f g f g w, T f f g w), d(\text { fgfgw, Sgfgw })\}
\end{aligned}
$$

From Equation (4) and Equation (6) we have

$$
\max \{d(\operatorname{Sgfg} w, T S f g w), d(f g g S w, S g g S w)\}<\max \{d(f g f g w, S g f g w), d(f g f g w, T f f g w)\}
$$

From Equation (5) and Equation (7) we have

$$
\max \{d(S T f g w, T f f g w), d(f g f T w, T f f T w)\}<\max \{d(\text { fgfgw,Tffgw }), d(\text { fgfgw, Sgfgw })\}
$$

If

$$
\max \{d(f g f g w, S g f g w), d(\text { fgfgw, Tffgw })\}=d(\text { fgfgw, Sgfgw })
$$

Then from Equation (8), fgfgw $\notin B_{g S w} \Rightarrow f g z \notin B_{g S w}$. Hence $B_{z} \not \subset B_{g S w}$. It is a contradiction to the maximality of $B_{z}$ in $A$, since $g S w \subseteq g f(X)=f g(X)$

If

$$
\begin{aligned}
& \max \{d(\text { fgfgw, Sgfgw }), d(\text { fgfgw,Tffgw })\} \\
& =d(\text { fgfgw,Tffgw })
\end{aligned}
$$

Then from Equation (9), fgfgw $\notin B_{f T w} \Rightarrow f g z \notin B_{f T w}$. Hence $B_{z} \not \subset B_{f T w}$.It is a contradiction to the maximality of $B_{z}$ in $A$, since $f T w \subseteq f g(X)$.

So

$$
f(g f g w) \in S(g f g w), g(f f g w) \in T(f f g w)
$$

In addition, $f(g f g w)=g(f f g w)$.

Using (b), (c) and Equation (10), we obtain

$H($ Sgfgw, Tffgw $)<\max \{d($ fgfgw, gffgw $)$,

$$
\begin{aligned}
& d(\text { fgfgw, Sgfgw }), d(\text { gffgw, Tffgw })\} \\
& =0
\end{aligned}
$$

Hence $S(g f g w)=T(f f g w)$.

Then the proof is completed.

Theorem 3.2 Let $(X, d)$ be an ultra metric space.
Let $T, S: X \rightarrow C(X)$ be a pair of multi-valued maps and $f: X \rightarrow X$ be a single-valued maps satisfying

(a) $f(X)$ is spherically complete;

(b) $H(S x, T y)<\max \{d(f x, f y), d(f x, S x), d(f y, T y)\}$ for all $x, y \in X$, with $x \neq y$;

(c) $f S=S f, f T=T f, S T=T S$;

(d) $S(X) \subseteq f(X), T(X) \subseteq f(X)$.

Then $f, S$ and $T$ have a coincidence point in $X$.

Moreover, if $f$ and $S, f$ and $T$ are coincidentally commuting at $z \in C(f, T)$ and $f f z=f z$, then $f, S$ and $T$ have a common fixed point in $X$.

Proof. If $f=g$ in Theorem 2.1, we obtain that there exist points $u$ and $v$ in $X$ such that

$$
f u \in S u, f v \in T v, f u=f v, S u=T v .
$$

As $u \in C(f, S), f$ and $S$ ipipare coincidentally commuting at $u$ and $f f u=f u$.

Write $w=f u$, then $w \in S u, w \in T v$.

Then we have

$$
f w=w
$$

and

$$
w=f w \in f(S u) \subseteq S(f u)=S w .
$$


Now, since also $u \in C(f, T), f$ and $T$ are coincidentally commuting at $u$ and $f f u=f u$, so we obtain

$$
w=f w \in f(T v) \subseteq T(f v)=T w .
$$

Thus, we have proved that $w=f w \in S w \cap T w$, that is, $w$ is a common fixed point of $f, S$ and $T$.

Corollary 3.3 Let $(X, d)$ be a spherically complete ultra metric space. Let $T, S: X \rightarrow C(X)$ be a pair of multi-valued maps satisfying

(a) $H(S x, T y)<\max \{d(x, y), d(x, S x), d(y, T y)\}$ for all $x, y \in X$, with $x \neq y$;

(b) $S T=T S$.

Then, there exists a point $z$ in $X$ such that $z \in S z \cap T z$ and $S z=T z$.

Remark 1 If $S=T$ in Corollary 3.3, then we obtain the Theorem of Ljiljana Gajic [6].

Remark 2 If in Theorem 3.1, $S=T, f=g$, we obtain Theorem 9 of K. P. R. Rao at [7].

Remark 3 If $S$ and $T$ in Theorem 3.1 are single-valued maps, then: 1) we obtain the results of K. P. R. Rao [4]; 2) $S=T, f=g=I$, we obtain the result of Ljiljana Gajic [3]; 3) $S=T, f=g$, then, we obtain Theorem 4 of K. P. R. Rao at [7].

\section{Conclusion}

In this paper, we get coincidence point theorems and common fixed point theorems for two pair of multi-valued and single-valued maps satisfying different contractive conditions on spherically complete ultra metric space, which is generalized results of [3-7].

\section{Acknowledgements}

Foundation item: Science and Technology Foundation of Educational Committee of Tianjin (11026177).

\section{REFERENCES}

[1] A. C. M. van Roovij, "Non Archimedean Functional Analysis,” Marcel Dekker, New York, 1978.

[2] C. Petalas and F. Vidalis, "A Fixed Point Theorem in Non-Archimedaen Vector Spaces," Proceedings of the American Mathematics Society, Vol. 118, 1993, pp. 819821. doi:10.1090/S0002-9939-1993-1132421-2

[3] L. Gajic, “On Ultra Metric Spaces,” Novi Sad Journal of Mathematics, Vol. 31, No. 2, 2001, pp. 69-71.

[4] K. P. R. Rao, G. N. V. Kishore and T. Ranga Rao, "Some Coincidence Point Theorems in Ultra Metric Spaces,” International Journal of Mathematical Analysis, Vol. 1, No. 18, 2007, pp. 897-902.

[5] J. Kubiaczyk and A. N. Mostafa, "A Multi-Valued Fixed Point Theorem in Non-Archimedean Vector Spaces," Novi Sad Journal of Mathematics, Vol. 26, No. 2, 1996, pp. 111116.

[6] L. Gajic, "A Multivalued Fixed Point Theorem in Ultra Metric Spaces,” Matematicki Vesnik, Vol. 54, No. 3-4, 2002, pp. 89-91.

[7] K. P. R. Rao and G. N. V. Kishore, "Common Fixed Point Theorems in Ultra Metric Spaces,” Journal of Mathematics, Vol. 40, 2008, pp. 31-35.

[8] B. Damjanovic, B. Samet and C. Vetro, "Common Fixed Point Theorem for Multi-Valued Maps,” Acta Mathematica Scientia, Vol. 32, No. 2, 2012, pp. 818-824. doi:10.1016/S0252-9602(12)60063-0 